\title{
ORIGINAL RESEARCH

EFFECT OF TASKORIENTED EXERCISES ON STANDING
BALANCE IN SUBJECTS WITH STROKE

${ }^{1}$ B. Deekshita

${ }^{2}$ V. Srikumari

${ }^{3} \mathrm{~K}$. Madhavi

\section{ABSTRACT}

Background: Stroke is an acute onset of neurological dysfunction resulting in the most common disability. Balance among these stroke subjects is impaired. To determine the effect of task oriented exercises on standing balance in subjects with stroke using performance oriented tinetti assessment score and single leg stance time is the need if the study.

Methods: Participants were taken from outpatient department of physiotherapy and neurology department in the institute. Randomized controlled trial with Prospective experimental design with simple random sampling including 30 subjects. An inclusion criterion includes stroke subjects with age 40- 75, both genders who were confirmed as stroke subjects by MRI n CT scan investigations. Right or left hemiplegia who are conscious and coherent were included. Subjects with spinal injuries, cognitive, perceptual disorders, shoulder subluxations, cardiac problems were excluded. Participants received daily 45 minutes of task oriented exercises protocol for 6days a week for 6weeks for study group and conventional physiotherapy for control group.

Results: Performance oriented tinetti assessment score and Single leg stance time which is valid and reliable measure for balance in stroke subjects was used. There is a significant improvement in performance oriented tinetti assessment score and single leg stance time from baseline to the end of intervention with significance $(\mathrm{P}<0.01)$ existing between the baseline and $6^{\text {th }}$ week in both variables.

Conclusion: Task oriented exercises appeared effective in improving standing balance in subjects with stroke. Single leg stance duration improved on task oriented exercise approach.

Keywords: Stroke, standing balance, task oriented exercises, single leg stance.

Received $21^{\text {th }}$ July 2014, revised $01^{\text {st }}$ October 2014, accepted $06^{\text {th }}$ October 2014

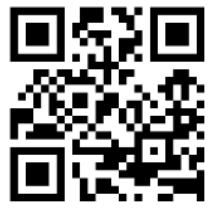

Www.ijphy.org
DOI: 10.15621/ijphy/2014/v1i5/55273
${ }^{2}$ Assistant Professor,

Sri Venkateswara Institute of Medical Sciences, (SVIMS), Tirupati.

${ }^{3}$ Principal,

Sri Venkateswara Institute of Medical Sciences,

(SVIMS), Tirupati.

\section{CORRESPONDING AUTHOR}

\section{${ }^{1}$ B. Deekshita}

MPT (Neurology)

College of Physiotherapy,

Sri Venkateswara Institute of Medical

Sciences, (SVIMS)

Tirupati. 


\section{INTRODUCTION}

Stroke is an acute onset of neurological dysfunction due to an abnormality in cerebral circulation with resultant signs and symptoms that corresponds to involvements of focal areas of brain. ${ }^{1}$ The WHO rates this as the third biggest health problem with India the second highest incidence rate in the world. The incidence of the disease in India to be around 130 per 100,000 population every year and says about $20 \%$ of heart patients are susceptible to it. $^{2}$

The disease has turned out to result in most common disability and dependence with more than $70 \%$ of stroke survivors remaining vocationally impaired and more than 30\% requiring assistance with activities for daily living. ${ }^{3}$ The resulting paralysis after stroke reduces motor skills and related functional abilities. After stroke, the recovery of balance is considered to be of crucial importance to achieve autonomy in activities of daily living ${ }^{4}$. Both static and dynamic balance can be improved by various physiotherapy treatment approaches. Several techniques have been developed to improve daily living activities like sitting, standing and walking without assistance devices. ${ }^{5}$

Balance impairment after stroke results in loss of static and dynamic balance with decreased proprioception, somatosensory and vestibular function. Balance re-education is very important in post stroke rehabilitation. ${ }^{6}$ Through specific physical exercises, balance re-reduction enables patients to regain or improve balance and better control over activities of daily living. Therapists are using different exercise modalities such as balance board, sit-fits, Swiss ball therapy, and individually designed exercises to help patients to improve balance and physical wellbeing as well. ${ }^{7,8}$

Task oriented exercises are very helpful in improving balance and activity levels of the patients especially after stroke. These exercises have been used for quite a long time by physiotherapists as an effective means of improving balance. ${ }^{9}$ Several researchers have experimented various surfaces in training balance to add to the effectiveness of task oriented exercises. ${ }^{10}$ This approach includes a wide range of interventions such as treadmill training, walking training on the ground, bicycling programmes, endurance training and circuit training, sit-to-stand exercises, and reaching tasks for improving balance. In addition, use is made of arm training using functional tasks such as grasping objects, and mental imagery. ${ }^{11,12}$

Present study uses task oriented exercises to improve standing balance in subjects with post stroke. The decreased ability to maintain static and dynamic balance after stroke could be related to the inability to select reliable sensory information (visual, vestibular, somatosensory systems) Exercise interventions, in the form of task-oriented exercise programmes, are now recognized as a new strategy to improve the functional status of stroke individuals. ${ }^{13}$

\section{MATERIALS AND METHODOLOGY}

Task oriented exercises is a new strategy to improve static and dynamic balance after stroke and improve process of somatosensory integration. It is used to stimulate awareness and proprioception, balance, coordination. ${ }^{14,15}$ Aim of the study is to evaluate the effectiveness of task oriented exercises on standing balance in subjects with stroke. Subjects were recruited from the department of physiotherapy OP, general ward and neurology OPD of the institute. A randomized controlled trail, prospective experimental design with pretest- post test design. Total 30 subjects with 15 in each group fulfilling inclusive criteria which includes stroke subjects with age between 40-75 years, both male and female with both right and left hemiplegic hemorrhagic type between 1 week to 3 months from onset of stroke who are conscious coherent with no other comorbidity Stroke subjects able to stand with tinetti balance score $<10$.Stroke subjects with cardiopulmonary dysfunctions, shoulder subluxation, poor GCS orthopaedic surgeries (TKR/THR) were excluded.

\section{Procedure:}

Participants are provided with full explanation and information sheet, written consent was taken from them after going through the information sheet. Information to the relevant medical team was given and suggestions were accepted for each individual participant .Data was collected over 6 weeks the lead researcher recorded all outcomes and provided all therapy except during the period of absence, when an alternate researcher covered this.

Baseline recordings of tinetti balance score and single leg stance time were taken from participants before the intervention by lead researcher twice and mean was taken as baseline. 
CONSORT PICTURE

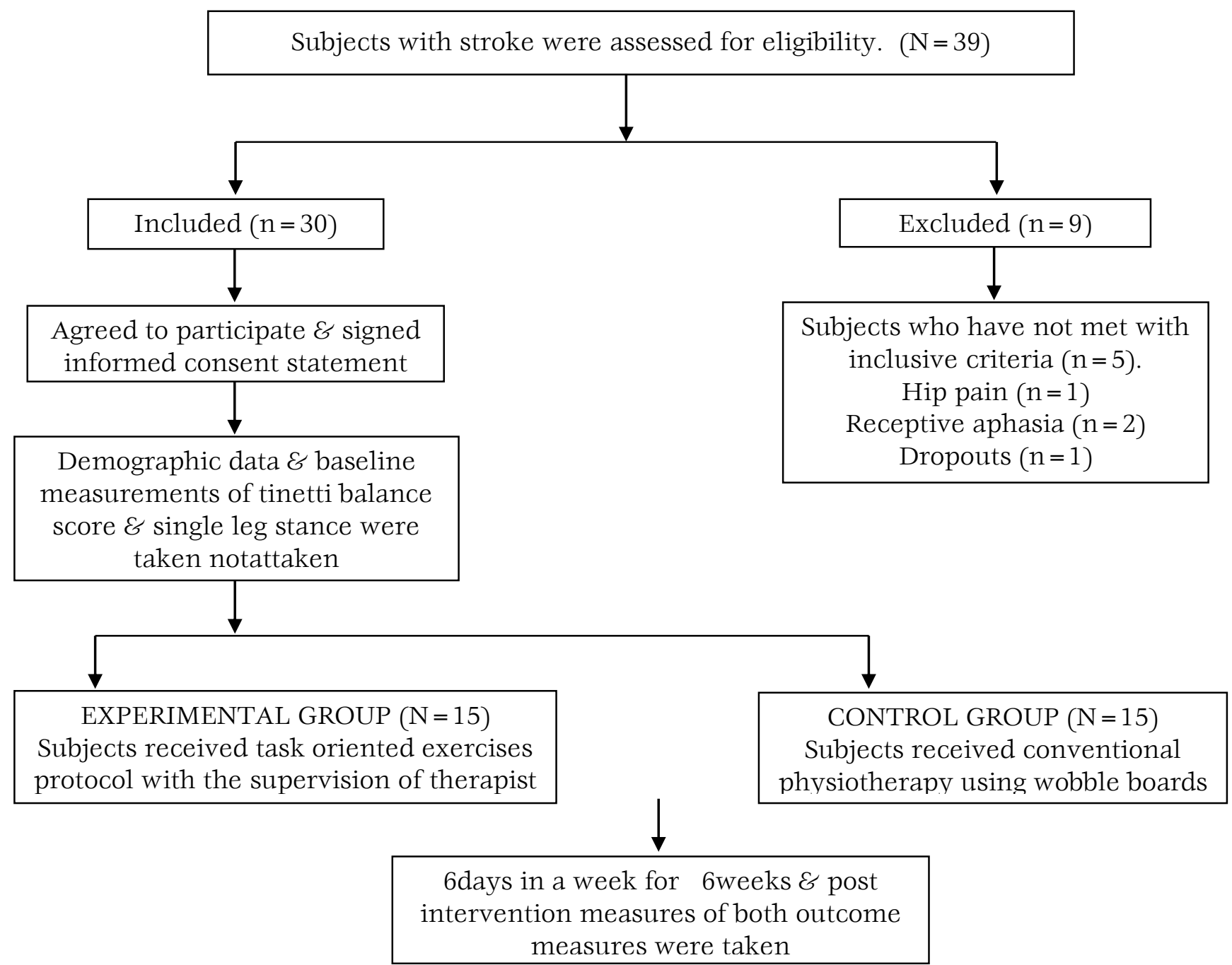

Table - 1 Demographic data of the subjects in both experimental and control groups

\begin{tabular}{|c|c|c|c|}
\hline variables & $\begin{array}{l}\text { Experimental } \\
\text { group } \\
(\text { mean }+/-S D)\end{array}$ & $\begin{array}{l}\text { Control group } \\
(\text { mean +/-SD) }\end{array}$ & $\begin{array}{l}p \text {-value } \\
\text { (2 tailed) }\end{array}$ \\
\hline Age & $49.57+/-9.39$ & $51.73+/-6.37$ & 0.281 \\
\hline Sex M:F & $9: 6$ & $11: 4$ & $X^{2}=3.595, p=0.084$ \\
\hline Height (cm) & $157.93+/-2.537$ & $157.53+/-3.218$ & 0.48 \\
\hline
\end{tabular}

Intervention phase began immediately after baseline and lasted for 6weeks, lead researcher was provided with task oriented protocol. Treatment protocols were designed by the lead researcher after discussing with the senior neurophysicaltherapist, and they were unique and changes every week according the participants standing recovery, acceptance level. Intervention was given to experimental group with informed consent. It includes stepping forward, backward and sideways. Stepping over blocks of various heights. Standing up from a chair, walking 4 steps forward, performing bilateral stool touch and walking backwards to the chair. Standing up from chair, walking 4 steps forward, turning to right, stepping over the exercise step. Turning to right again and walking forwards to chair. (repeat circuit in opposite direction) Performing double leg stance for $10 \mathrm{sec}$. Performing tandem stance for $10 \mathrm{sec}$. Rising from chair without using arms. Walking forward and backward with tandem walking. Performing single leg stance for $10 \mathrm{sec} .45 \mathrm{~min}$. per day, 6days per week, 6wks. For experimental group and conventional physiotherapy for control group.

\section{RESULTS}

The following outcomes were measured weekly throughout the intervention phase of the study to record performance. Performance oriented tinetti 
balance score with total score ranges from 0-24. Tinetti Assessment Tool mainly assesses body structure (impairments), but Tinetti Balance Test evaluates activities (limitations to activitydisability): mobility $=$ changing $\&$ maintaining body position. Single leg stance time is one of the most challenging gauges of stability while standing on a narrow area of support" and averred it to be the most frequently used measure of balance I $n$ physical training studies involving older adults. It has reliability and is a valid standing assessment tool. Primarily data was analyzed by trend (direction in which the series of score is progressing). Change scores were calculated as the difference between baseline scores and the maximal scores attained in the intervention Conformation of visual interpretation of data is enhanced by conforming through statistical analysis. To observe the statistical significance as well as the trend between baseline and $6^{\text {th }}$ week paired samples $t$ test has been performed for the variables tinetti balance score and single leg stance time separately. To verify whether there is a trend in both variables curve fitting technique is applied. All 30 participants with 15 in each experimental and 15 in control group completed all phases based on the respective group protocols with no adverse effects. This was considered to be a positive result. Tinetti balance scores and total change scores for each participant. Improvement occurred in all the 30 participants from $1^{\text {st }}$ week to $6^{\text {th }}$ week in both experimental and control groups. Improvements in scores differed in both groups. In experimental group change of scores ranged from 7 to 13.6 ( mean). In control group change of scores ranged from 6.4 to 9.06 (mean)

Table-2 Analysis of tinetti balance score in pre and post intervention in experimental group

\begin{tabular}{|c|c|c|c|c|c|c|c|}
\hline \multirow{2}{*}{ Group } & parameter & & N & mean & $\begin{array}{c}\text { Standard } \\
\text { deviation }\end{array}$ & t-value & p-value \\
\hline \multirow{2}{*}{ experimental } & $\begin{array}{c}\text { Performance } \\
\text { oriented tinetti } \\
\text { balance }\end{array}$ & pre & 15 & 7 & 1.851 & 18.33 & $<0.05$ \\
\hline
\end{tabular}

Table-3 Analysis of tinetti balance in control group

\begin{tabular}{|c|c|c|c|c|c|c|c|}
\hline Group & Parameter & & $\mathbf{N}$ & mean & $\begin{array}{l}\text { Standard } \\
\text { deviation }\end{array}$ & t-value & p-value \\
\hline \multirow{2}{*}{ control } & \multirow{2}{*}{ Tinetti balance } & pre & 15 & 6.4 & 1.404 & \multirow{2}{*}{12.64} & \multirow{2}{*}{$<0.05$} \\
\hline & & post & 15 & 9.06 & 1.162 & & \\
\hline
\end{tabular}

The total Single leg stance timescores across the baseline and intervention showed a positive change in trend in all participants.all showed a period of latency before change in total scores was evident, these periods of latency ranged from $1^{\text {st }}$ week to $3^{\text {rd }}$ week.Improvements were seen in all subsections for 30 participants.

Table-4 Analysis of single leg stance in pre and post intervention in experimental group

\begin{tabular}{|c|c|c|c|c|c|c|c|}
\hline Group & parameter & & $\mathbf{N}$ & mean & $\begin{array}{l}\text { Standard } \\
\text { deviation }\end{array}$ & t-value & p-value \\
\hline \multirow{2}{*}{ experimental } & \multirow{2}{*}{$\begin{array}{c}\text { Single leg } \\
\text { stance }\end{array}$} & pre & 15 & 6.53 & 2.263 & \multirow{2}{*}{22.52} & \multirow{2}{*}{$<0.05$} \\
\hline & & post & 15 & 14.6 & 2.995 & & \\
\hline
\end{tabular}

Table-5 Analysis of single leg stance in control group

\begin{tabular}{|c|c|c|c|c|c|c|c|}
\hline Group & parameter & & $\mathbf{N}$ & mean & $\begin{array}{l}\text { Standard } \\
\text { deviation }\end{array}$ & t-value & p-value \\
\hline \multirow{2}{*}{ control } & \multirow{2}{*}{$\begin{array}{l}\text { Single leg } \\
\text { stance }\end{array}$} & pre & 15 & 6 & 1.690 & 6.32 & \multirow{2}{*}{$<0.05$} \\
\hline & & post & 15 & 8.6 & 1.877 & & \\
\hline
\end{tabular}


Table-6 Between the groups post intervention scores

\begin{tabular}{|c|c|c|c|c|}
\hline & groups & N & Mean & Std. Deviation \\
\hline $\begin{array}{c}\text { TINETTI BALANCE } \\
\text { ASSESMENT }\end{array}$ & Experimental & 15 & 6.4 & 1.35225 \\
\hline & control & 15 & 2.6667 & 0.8165 \\
\hline SINGLE LEG STANCE & Experimental & 15 & 8.0667 & 1.38701 \\
\hline & control & 15 & 2.6667 & 1.63299 \\
\hline
\end{tabular}

\section{Graph-1}

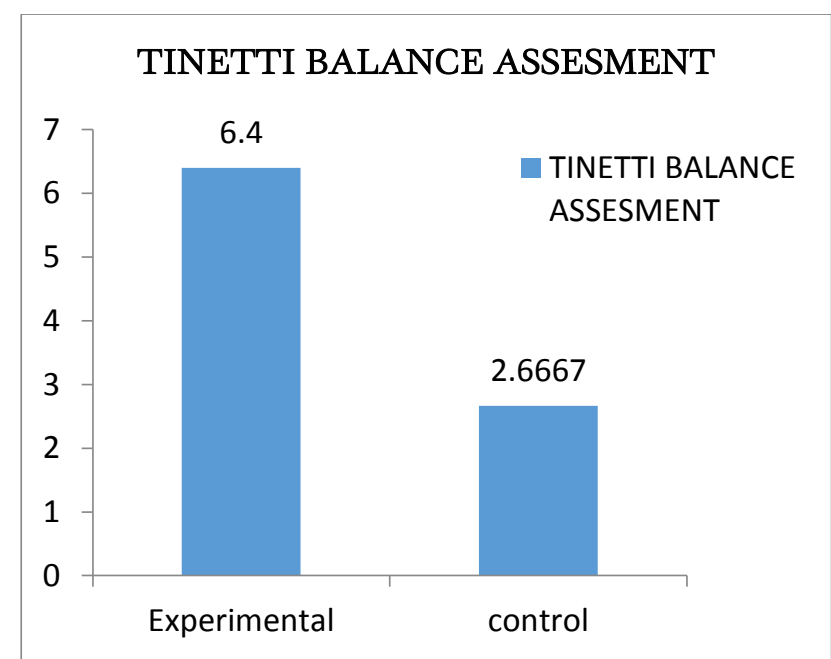

\section{Graph-2}

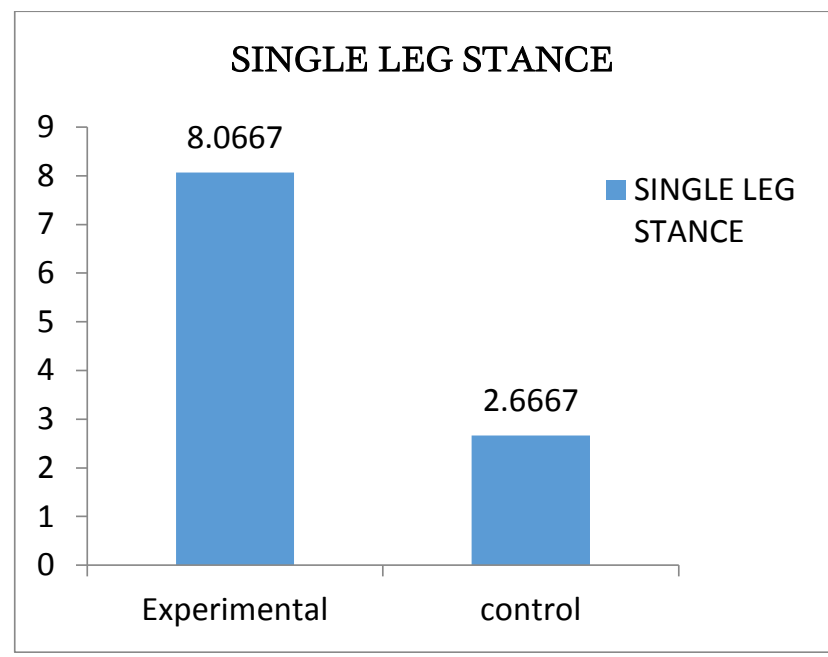

\section{DISCUSSION}

This study has explored the effects of task oriented exercises on standing balance in the sub-acute phase of rehabilitation after stroke. We found improvement in standing balance and performance of activity in 30 participants, with clear difference in total single leg stance time and performance oriented tinetti balance scores between baseline and $6^{\text {th }}$ week of intervention. Even though risk for mortality is more in ICH stroke clients, there is an evidence of good prognosis when compared to the infarct type of stroke, if the resolution of hematoma occurs early after ictus. Risk of mortality increases in ICH associated with any intra ventricular bleed, poor GCS and midline shift.

The significant results of the current study are may be due to absence of factors responsible for poor prognosis. Other factors responsible for significant results are early recruitment of participants into intervention after ictus i,e., ranged from 2-10 weeks after ictus. Due to early recruitment after ictus task oriented exercises has enhanced standing balance by minimizing the unbalanced forces and adapting better stability to perform activity.

Even though the baseline scores of performance oriented tinetti balance showed least scores in few participants, showed better results along with other participants after intervention. Single leg stance time improved along with balance and stability. Feber MJ et al in their studies, stated that Performance Oriented Mobility Assessment (POMA) measures both static and dynamic balance using tasks testing balance and gait

The current study supports the early hypothesis which states significant proprioception and somatosensory ${ }^{54}$ information to brain, facilitating direct brain activation to maintain standing balance. As a result it is evident that task oriented exercises activate the standing balance and the ability to perform activity with good stability.

Katz-Leurer et al. in his two studies performed with patients in the chronic phase has shown. In one study that the effects on stroke survivors of a task-oriented exercise programme were compared with altered sensory input.

Pollock et al in his study, three small studies of good quality focusing on task-oriented exercises and using functional outcome measures were identified and reviewed.

In the present study scores of performance oriented tinetti balance showed latency in improving scores. Latency in scores was proportional to the severity in balance impairment. Better scores were noted in the experimental group rather than the control after the intervention. This signifies the effectiveness of task oriented 
exercises in improvising the standing balance and performance.

Statistical results signify the differences in the performance oriented scores at $6^{\text {th }}$ week showed better results in the intervention group than the control group. Ability to perform activity was better in the intervention group

\section{CONCLUSION}

The study for 6 weeks using task oriented exercises appeared to be effective in improving standing balance and performance of activity in subjects with stroke. The latency for change in the improvement changes with the severity in balance impairment. Recovery was noted better in single leg stance time with the participants in the intervention group compared to control group. Task oriented exercises is a focused intervention in improving the ability to perform a task.

Quality of life was better in the intervention group after $6^{\text {th }}$ week than the control group. Tinetti balance scores showed better results from baseline to $6^{\text {th }}$ week. Task oriented exercises appeared effective in improving standing balance in subjects with stroke. Single leg stance duration improved on task oriented exercise approach. Intervention is beneficial in improving standing balance in stroke subjects.

\section{REFERENCES}

1. Kathlen strong,colin mathers, ruth bonita. Preventing stroke: saving lives around the world. Lancet Neurol. 2007;6(2):182-7

2. Banerjee $\mathrm{T}$, dass. Epidemiology of stroke in India. Neurology Asia 2006; 11: 1 - 4.

3. FionaC Taylor, suresh kumarK. stroke India fact sheet ( updated 2012).

4. FeiginV, LawesC,BennetCelloS, ParagV. Worldwide stroke incidence and early case fatality in 56 population based studies; a systemic review. Lancet neurol. 2009; 8(4): 355369.

5. Susan B.O' Sullivan, Thomas J. Schmity. Physical rehabilitation. $5^{\text {th }}$ edition; 2007.

6. A $\mathrm{m}$ bronstein, $\mathrm{t}$ brandt. Clinical disorder of balance disorders, gait and posture. J Neurol Neurosurg Psychiatry. 1997; 63:415.

7. David Good. Handbook of Neurorehabilitation. $1^{\text {st }}$ Edi; 1994.

8. Dean CM, Shepherd RB. Task- related training improves performance of seated reaching tasks after stroke, A randomized controlled trial. Stroke.1997; 28(4); 722-8.

9. Raymond D Adams, Maurice Victor, Allan H. Ropper. Principles of Neurology, 6 $6^{\text {th }}$ Edi; 1997.

10. Ottawa Panel, Khadilkar A, Phillips K, et al, Ottawa Panel. Evidence-based clinical practice guidelines for post-stroke rehabilitation; Top Stroke Rehabil. 2006 Spring; 13 (2); 1-269.

11. Chen IC, Cheng PT, Chen CL, et al. Effects of balance training on hemiplegic stroke patients. Chang Gung Med J.2002; 25(9): 583-90.

12. Duncan P, Studenski S, Richards L, et al. Randomized clinical trial of therapeutic exercise in sub acute stroke. Stroke. 2003; 34(9):2173-80.

13. Bayouk, Jean-Francois A, Boucher, et al. Balance training following stroke: effects of task oriented exercises with and without altered sensory input. International Journal of Rehabilitation Research. 2006; 29(1):51-59.

14. Jang $\mathrm{SH}$, Kim $\mathrm{YH}$, Cho $\mathrm{SH}$, et al. Cortical reorganization induced by task- oriented training in chronic hemiplegic stroke patient. Neuroreport. 2003;14(1):137-41.

15. Lehman GJ, Hoda W, Oliver S. Trunk muscle activity during bridging exercises on and off a Swiss ball. Chiropractic \& Osteopathy. 2005; 13:14.

\section{Citation}

B. Deekshita, V. Srikumari \& K. Madhavi. (2014). EFFECT OF TASKORIENTED EXERCISES ON STANDING BALANCE IN SUBJECTS WITH STROKE. International Journal of Physiotherapy, 1(5), 242247. 